Research Article

\title{
Mobile Information System of Ceramic Appreciation and Communication Management Based on Mobile Edge Computing
}

\author{
Mian Wang \\ Department of Arts and Crafts, Lu Xun Academy of Fine Arts, Shenyang 110004, Liaoning, China \\ Correspondence should be addressed to Mian Wang; wangmian@lumei.edu.cn
}

Received 14 July 2021; Revised 12 August 2021; Accepted 31 August 2021; Published 16 September 2021

Academic Editor: Sang-Bing Tsai

Copyright ( 12021 Mian Wang. This is an open access article distributed under the Creative Commons Attribution License, which permits unrestricted use, distribution, and reproduction in any medium, provided the original work is properly cited.

\begin{abstract}
Mobile edge computing is a very popular technology now. It was proposed to eliminate the problem of lack of global computing resources. This article aims to study the use of the latest mobile edge computing technology to study the mobile information system for appreciation, exchange, and management of the traditional ceramic industry. The whole article uses mobile edge computing technology. It enters the network using wireless methods and provides recent users with the required services and cloud computing functions, allowing users to easily query the information and data they want, plus mobile. The information system enables people to use mobile phones, tablets, and other mobile terminals to query information in the ceramic industry and perform functions such as appreciation, communication, and management. From 2016 to 2020, our country's ceramic industry exports have increased from US $\$ 3.067$ billion to US\$6.826 billion. Traditional ceramics in our country have been loved by various industries at home and abroad. The number of employees in the ceramic industry has also increased to 5 million, an increase of $30 \%$ year-on-year. The ceramic industry is also very promising in the long term.
\end{abstract}

\section{Introduction}

Since the beginning of the 21st century, the increasing number of mobile devices new services, like 3D video games and face recognition that require higher processing capabilities of mobile terminals, is developing rapidly, and the management of mobile terminals will face huge challenges. Currently, MEC technology is used to solve the lack of global computing resources due to high mobile accessibility. In order to cope with the next generation of $5 \mathrm{G}$ mobile communications, mobile network traffic has become more expensive, with higher performance, and the application complexity is not limited by the terminal. The MEC is designed and developed to transfer the computing load from the main cloud data center to the mobile equipment to reduce network pressure. In the traditional radio access network (RAN), the base station is deployed at the edge of the mobile network and can switch services. However, the base station will not analyze or respond to user requests. Therefore, traditional base stations cannot provide edge computing services beyond network connections.
Until now, in order to solve the problems of the rapid development of the mobile Internet and the development of modern wireless networks, small base stations with certain computing and storage capabilities have been located, and the small base stations have been placed on the edge of the large mobile Internet to accelerate the speed of interconnection. The purpose of solving the above problems provides users with lower latency and higher bandwidth and reduces the burden on the backbone network. Therefore, the ETSI provides MEC as a model, a promising computing model. The basis of the model is the evolution of $5 \mathrm{G}$ networks and is a proposed emerging technology for the deep integration of Internet services and small cells. The carrier node usually consists of a series of small or very small base stations with computer and storage capabilities. It is also used at the edge of the network to enable mobile handheld devices, terminals, and very close components to provide processing and storage services to meet the needs of higher computing workloads and low-latency processing, as well as reduce the workload of the backbone network. 
MEC is a new form of computation, storage, and distribution of network resources within a mobile radio access network (RAN), being environmentally sensitive and user friendly. This method reduces the burden on the backhaul and core network and is essential for realizing low-latency, high-bandwidth, and agile mobile services. Tran $\mathrm{T} X$ envisions a real-time, context-aware collaboration framework, which is located at the edge of the RAN, composed of MEC servers and mobile devices, and integrates heterogeneous resources at the edge. Specifically, Tran T X introduced and studied three powerful use cases, including mobile edge orchestration, collaborative caching and processing, and multilayer interference cancellation. We demonstrated the great advantages of these methods in facilitating the evolution to $5 \mathrm{G}$ networks. Finally, we discussed the key technical challenges and open research issues that need to be resolved in order to effectively integrate $\mathrm{MEC}$ into the $5 \mathrm{G}$ ecosystem. However, he did not do specific experiments and related data and provided a practical data support for the collaborative framework he envisioned [1]. Under dynamic pressure, the Internet has become one of the most important technologies in the international education industry and industry. However, a lot of small data from users is very scattered and not good for compression. This facilitates the use of computing resources and reduces the chance of accidental access. Additions are also very sensitive to delays in the transfer of user data, such as control data. However, traditional internet streaming does not always meet that requirement. The integration of edge mobile computing makes the Internet of Things easy for data retrieval, processing, management, and accessibility. In this piece, Liu L proposed a new Internet power system to increase the likelihood of accidental crashes and reduce data transfer rates. This consumes a lot of internet power data. Statistical results show that this architecture can increase the life expectancy and reduce transmission delays without increasing energy consumption. However, it does not take into account the current state of the art technology and may not meet the intended regulatory requirements [2]. Reducing overall power consumption and network latency is one of the most attractive issues for cloud computing (MCC) compliance and large-scale service level agreement (SLA). However, the delay caused by this loading and unloading process leads to the use of servers (called cloudlets) located close to the actual user. It allows what is known as mobile edge computing (MEC) to meet users' requirements for different types of services. And over a wide geographical area, Xiaoyun works by sending user requests from one small cloud to another. This type of collaboration affects energy consumption and latency. In this project, Jararweh et al. proposed an optimization model for MEC systems, taking these two points into account simultaneously. Specifically, Jararweh et al. looked at cloud sizes. There are two types of small clouds: local small clouds and global small clouds, which have higher capabilities. The user connects to the local cloudlet and sends all the traffic to it. If the local cloudlet cannot serve the request, the request will be transferred to the cloud on another machine. If there is no local cloudlet capable of serving the request, go to a central cloudlet that can provide all kinds of services. The Xiaoyun hierarchical network request process increases power consumption and latency. Our model reduces energy consumption while producing acceptable levels of lag. We evaluate the results in many real situations, a scenario to show that it can be used for real power optimization of large MEC systems without violating the delay constraints. A design model was applied to the MEC system, but no specific experiments were performed to design a detailed model of its design [3].

This paper uses the latest MEC, that is, mobile edge computing technology, for the traditional ceramic industry to develop and design a mobile information system for ceramic appreciation, communication, and management, so that people can query the information they want on the system at any time. The system architecture of mobile edge computing can allow nearby users to access the network and provide them with the services they need. Nowadays, the demand for computing and storage resources for end-to-end applications continues to increase, which will increase the workload of uninstalling mobile terminals in the cloud and become the most effective way to solve the problem of insufficient terminal performance. The integration of this technology with the ceramic industry can inject new vitality into the traditional industry. It has its own place in this fastdeveloping era and can well show our country's traditional culture.

\section{Mobile Edge Computing and Communication Management Mobile Information System}

2.1. Mobile Edge Computing. Mobile edge computing can easily access the network wirelessly and provide the latest telecommunications users with what they need, services and cloud computing functions [4]. It allows users to experience a very high-quality network experience, such as speeding up network loading, service response, application downloading, and other functions. As mobile devices continue to be created, a variety of products emerge in an endless stream. At this time, it is necessary for the computing power of mobile terminals to keep up with the needs of a large number of users, plus 5G, face recognition, VR games, and other technologies. This is a very serious problem for the development of all walks of life, and mobile edge computing technology emerged at the historic moment. It can well solve a large number of computing problems brought about by the massive access of next-generation mobile communication devices and can eliminate the problems of high mobile network costs, low efficiency, and multiple terminal applications $[5,6]$. Specifically as shown in Figure 1, MEC specific architecture enables nearby users to access the network and provide them with the services they need.

Clustering algorithm is an algorithm for studying specific classification problems. It is also an important algorithm for data mining. It is usually composed of several different models. It is widely used in autonomous learning. The defined data set can be specified and divided into multiple subsets, such as groups. In general, clustering is the process of splitting an instance into a set of data into independent subsets using an algorithm [7]. This particular 


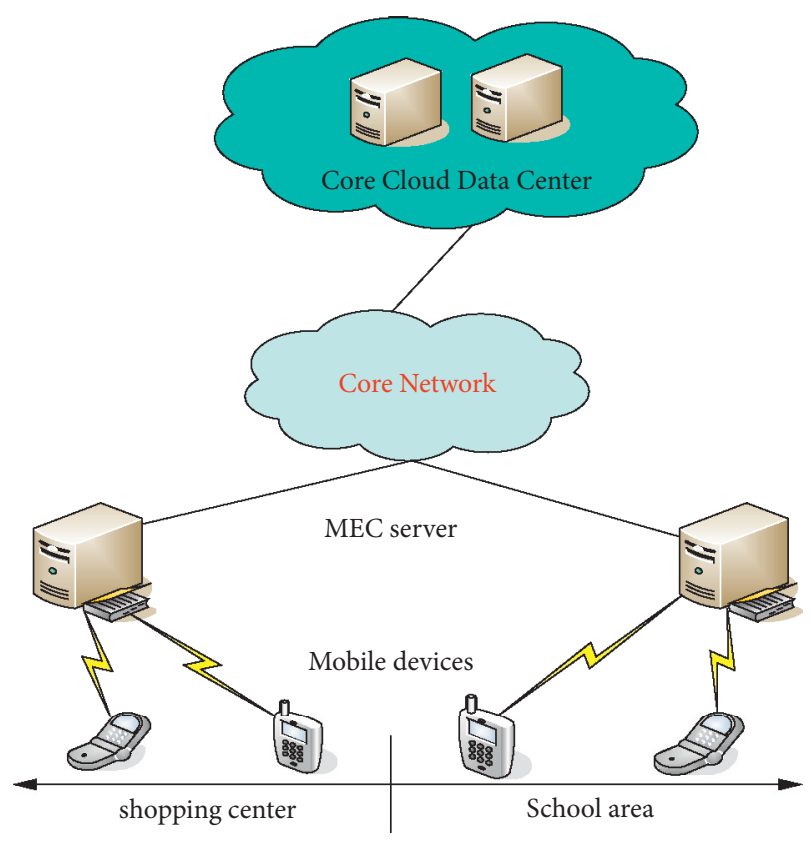

Figure 1: Schematic diagram of the MEC system architecture.

algorithm is to arrange the data sets to create a data cluster, and meet the following two conditions: 1 . subsequent tasks in the same cluster may be similar; 2 . the gap between the different groups of jobs should be as large as possible. There are two types of Euclidean distance and Mahalanobis distance in the clustering algorithm. The Euclidean distance formula can be expressed as

$$
c_{A, B}=\sqrt{\sum_{n=1}^{n}\left(A_{m}-B_{n}\right)^{2}} .
$$

The Mahalanobis distance can be expressed as

$$
c_{A, B}=\sum_{m=1}^{n}\left|A_{m}-B_{m}\right| .
$$

Ming's distance is an extension of Mahalanobis distance:

$$
c_{x, y}=\left(\sum_{i=1}^{n}\left|x_{i}-y_{i}\right|^{p}\right)^{1 / p} \text {. }
$$

It is also often used. It can be seen that, for the Ming's distance, when $p=1$, it corresponds to the Mahalanobis distance, and when $p=2$, it corresponds to the Euclidean distance [8].

Assume that, at a certain moment, the task in the SDN controller is $\left\{S_{1}^{*}, S_{2}^{*}, \ldots, S_{n}^{*}\right\}$, and the corresponding delay sensitivity is $\left\{P_{1}^{\prime}, P_{2}^{\prime}, \ldots, P_{n}^{\prime}\right\}$, respectively. After normalizing the delay sensitivity, the delay sensitivity obtained is $\left\{P_{1}^{*}, P_{2}^{*}, \ldots, P_{\mathrm{n}}^{*}\right\}$ respectively, where

$$
P_{i}^{*}=\frac{P_{i}^{\prime}}{\sum_{j=1}^{n} P_{j}^{\prime}} .
$$

The total amount of calculation consumed to complete the task is, respectively, $\left\{L_{1}^{\prime}, L_{2}^{\prime}, \ldots, L_{n}^{\prime}\right\}$ and the total calculation amount obtained after the normalization operation of the calculation amount is $\left\{P_{1}^{*}, P_{2}^{*}, \ldots, P_{n}^{*}\right\}$, where

$$
P_{i}^{*}=\frac{L_{i}^{\prime}}{\sum_{j=1}^{n} L_{j}^{\prime}} .
$$

Euclidean distance between jobs is $S_{i}^{*}, S_{j}^{*}$ in the same cluster which can be expressed as

$$
d_{i, j}=\sqrt{\left(P_{i}^{*}-P_{j}^{*}\right)^{2}+\left(L_{i}^{*}-L_{j}^{*}\right)^{2}} .
$$

The average of its values in the same cluster is

$$
\bar{d}=\frac{\sum_{i=1}^{n} \sum_{j=1, j \neq i}^{n} d_{i, j}}{C_{n}^{2}} .
$$

$D$ is the standard deviation in the work. When there is job $C_{i}$ in cluster $\left\{S_{1}^{*}, S_{2}^{*}, \ldots, S_{n^{\prime}}^{*}\right\}$, the corresponding standard deviation is

$$
D_{i}=\sqrt{\frac{\sum_{i=1}^{n^{\prime}} \sum_{j=1, j \neq i}^{n^{\prime}}\left(d_{i, j}-\bar{d}\right)^{2}}{C_{n^{\prime}}^{2}}} .
$$

We believe that, in the same complex, the lower the standard deviation of all tasks, the greater the similarity of the items. Therefore, we are waiting for the results of the merger. The maximum value of the standard deviation in all clusters is minimal [9]; that is, when all functions can be divided into $k$-blocks, we would expect

$$
\min \left\{\max D_{i}\right\}, \quad i=1,2,3, \ldots, k .
$$

2.2. MEC Server Technology. MEC servers can provide cloud services for mobile users over Wi-Fi hotspots, as shown in Figure 2. At present, computing and storage resource requirements are increasing among terminal applications on mobile terminals in the cloud. It has become the most effective solution to the problem of insufficient endpoint performance [10]. MEC servers bring the traditional cloud computing core to the edge of the network at the user side, including accelerating content, services, and applications. This will improve the edge response. Although MEC server provides users with a highly distributed computing environment, it can also perform additional functions that are not possible in traditional Internet architectures, such as real-time data analytics, and a better understanding of distributed cloud computing. For many emerging applications, such as video volume services and local streaming media services, when the local MEC server cannot meet the needs of users or the MEC server and the computing power of the mobile terminal, mobile providers can use the MEC server to work together with the main cloud [11]. The MEC server may request computing resources to complete the task. Small clouds that use MEC services have significant traffic delays. And the device with high Wi-Fi coverage is uninstalled and can work smoothly. MEC technology is 


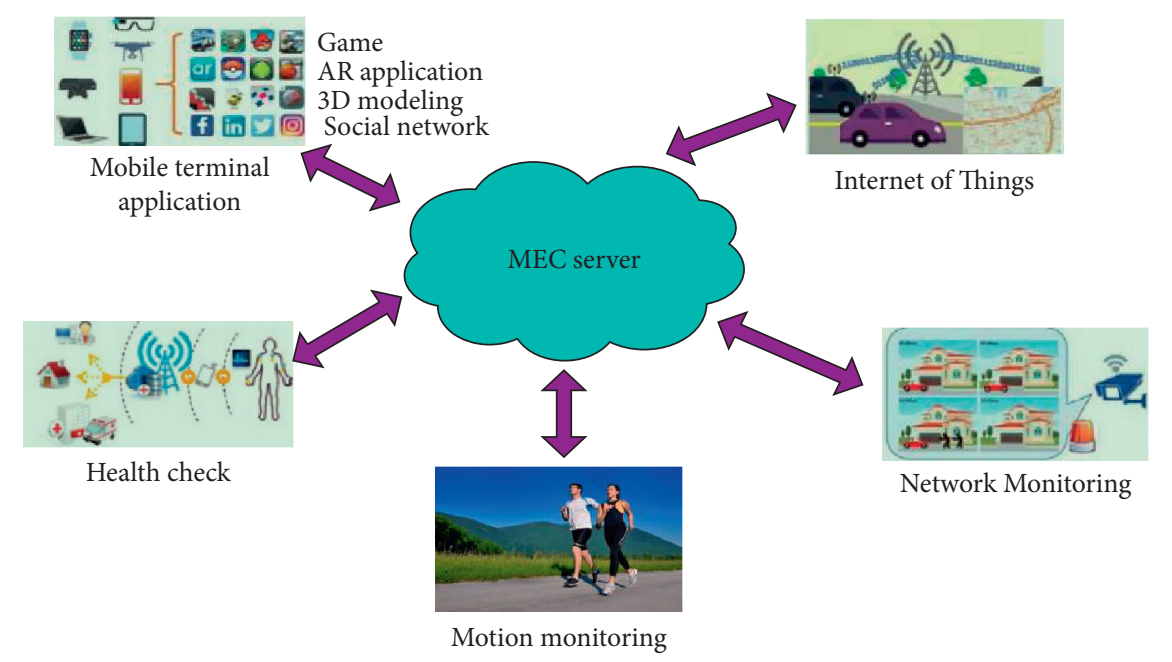

FIgURE 2: MEC system application scenario.

accredited by $5 \mathrm{GPPP}$ ( $5 \mathrm{G}$ infrastructure public private). It also includes virtual networks (NFV) and network resolution networks (SDN) [12].

When task $S_{i}$ is executed on the mobile terminal, the total system delay from issuing the uninstall request to the completion of functions can be specified as follows $t l_{i}$; when task $S_{i}$ is executed on the MEC server, the minimum total delay of the system can be expressed as

$$
\mathrm{TC}_{i}=\mathrm{Tt}_{i}+\mathrm{tb}_{i}+\min _{j \in \text { allMECserver }}\left\{\mathrm{Pc}_{i, j}+t_{i}\right\},
$$

where $\mathrm{Tt}_{i}$ represents the transmission delay, $\mathrm{tb}_{i}$ represents the waiting delay due to insufficient bandwidth, $\mathrm{Pc}_{i, j}$ represents the queuing delay on the MEC server $j$, and $t_{i}$ represents the task execution delay on the server. When task $S_{i}$ is executed on $j$, the execution delay is expressed as

$$
\mathrm{TC}_{i, j}=\mathrm{Tt}_{i}+\mathrm{tb}_{i}+\mathrm{Pc}_{i, j}+t_{i} .
$$

Assuming that the cost per unit time consumed when performing tasks and transmission tasks is 1 , the cost of consuming computing resources of mobile terminals and MEC servers to perform unit time tasks is $c_{\mathrm{ue}}$ and $c_{c}$ times the time cost [13], the cost defined in this article is an abstract concept; in the actual system, we can specifically define the dimensions according to the objects of interest, such as costs. Therefore, the cost of executing task $S_{i}$ on the mobile terminal is defined as

$$
u_{i, 0}=\mathrm{tl}_{i}+\mathrm{tl}_{i} \cdot c_{\mathrm{ue}} .
$$

The cost of performing task $S_{i}$ on the MEC server $\mathrm{MC}_{j}$ is

$$
u_{i, j}=\mathrm{TC}_{i, j}+t_{i, j} \cdot c_{c} \cdot
$$

In the single task mode, the local SDN server will choose the mobile terminal or MEC server with the least overhead to perform the task [14]; that is, when there are M MEC servers $\mathrm{MC}_{1}, \mathrm{MC}_{2}, \mathrm{MC}_{3}, \ldots, \mathrm{MC}_{M}$ in the cell, the task offloading strategy in the system can be expressed as

$$
j=\underset{j}{\arg \min }\left(u_{i, j}\right), \quad 0 \leq j \leq M .
$$

When $j=0$, the local SDN server rejects the uninstallation request of the task, and the task is executed on the mobile terminal.

From the STM mode, the total transmission delay $\mathrm{Tt}_{i}$ of task $S_{i}$ can be expressed as

$$
\mathrm{Tu}_{i}=\mathrm{Tt}_{i} .
$$

2.3. Mobile Information System. Mobile information refers to the use of integrated communication platforms including advanced mobile communication technology and mobile Internet technology, through the interactive data communication of multiple platforms such as terminals, to realize the mobilization of business and service management $[15,16]$. Generally speaking, mobile information is the realization of various information applications based on mobile terminals such as smart phones, tablets, smart watches, and technologies such as telecommunications and the Internet, and it finally allows mobile devices around you to be used for mobile at any time. The portability of servers and personal computers, the provision of information, electronization, and networking provide the society with efficient, high-quality, standardized, and transparent integrated management processes and services, and they can also interact with online information at any time. The mobile information system is one of the most popular mobile communication systems. It is a system designed with mobile information technology as the core, which can be integrated with all walks of life, such as finance, IT, sports, medicine, and other industries and lets you get information quickly and work anytime [17]. Compared with the traditional computer industry, the construction of the mobile information communication system is shown in Figure 3. It is more difficult and more demanding. It is the information construction of large institutions including governments, 


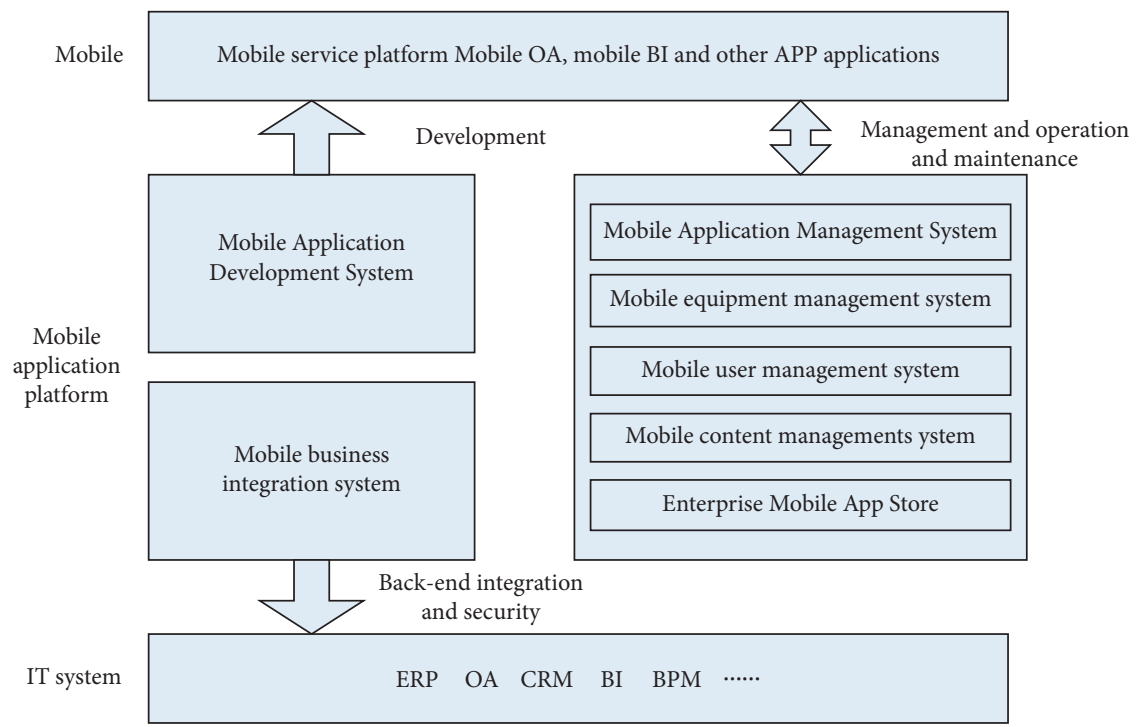

FIGURE 3: Structure diagram of the mobile information system platform.

enterprises, etc., which is very huge. The project needs to be gradually improved.

\section{Ceramic Appreciation and Communication Management Mobile Information System}

3.1. Hardware Design. The ceramic appreciation and communication management system studied in this article is based on mobile edge computing plus mobile information technology. Phone users can access networks through a variety of wireless sources, such as mobile terminals and $\mathrm{Wi}$ Fi access points. There are many deployments on the access site side. MEC server is closer to the user [18]. The technology that will be widely adopted in the future is to use relevant software in the mobile network to define the network infrastructure combined with NFV technology, such as H3C VNF Manager, VSR, vBRAS, and other software, which can be used to centrally control the SDN controller to realize the MEC system development and realization. According to requirements, the MEC system can be installed on one or more ordinary server equipment near the access site using NFV technology, or the MEC server can be directly integrated into the access site. An access site can directly communicate with multiple MEC servers through 1 to 2 data hops, and different user services can be forwarded to different MEC servers for editing. The same MEC server can be a multiaccess site [19]. To facilitate the analysis in this study, we found that data from the same job can only be forwarded to one MEC server for processing, and the same MEC server can only process data from one company at a time. New arrivals are placed in the work buffer queue of the MEC server, waiting to be processed, and the queue can be managed in a first-in, first-out manner [20].

3.2. Software Design. The business logic and functions of the ceramic appreciation and communication business management redesign process are optimized for the older business processes, mainly to improve the work of the initial art appraisal and art investment consulting companies [21]. In addition, a new business of artwork protection has been added to ensure that the artwork will not be damaged. The administrator provides the existing artwork to the system for storage, and after careful study and analysis by experts, it determines which set of artwork should be recognized and highlighted and can view pending audit requests, and experts appraise the age and art of the artwork value for verification. After the appraisal is completed, the expert submits the appraisal result online and there must be an identification structure, and another test will be done next time to confirm the correctness of the appraisal result [22]. After the evaluation, the management must submit and record the evaluation results. The process of evaluating art has not changed much from the traditional process. The main reason is that art lovers submit art evaluation requests online and managers accept the requests online and assign successful requests to designated experts [23]. First of all, the administrative staff will formulate an artwork protection plan. Next, select the artwork that needs to be protected and preserved to highlight it. The designated expert will formulate a protection plan based on the actual condition of the artwork. Finally, the personnel who take preventive and management measures will record the protection files on the system, as shown in Figure 4.

3.3. Mobile Information System. This article discusses in detail the mobile information system for ceramic appreciation and communication management, using mobile edge computing technology. Since ancient times, our country has collectively referred to pottery and porcelain as ceramics and it has been passed down [24]. Ceramics are made of highviscosity, high-elasticity clay as the main raw material. They are opaque, have small pores, have poor water absorption, and have a muddy sound. Porcelain made of clay, silver, and quartz in our daily life is translucent, is nonabsorbent, is 


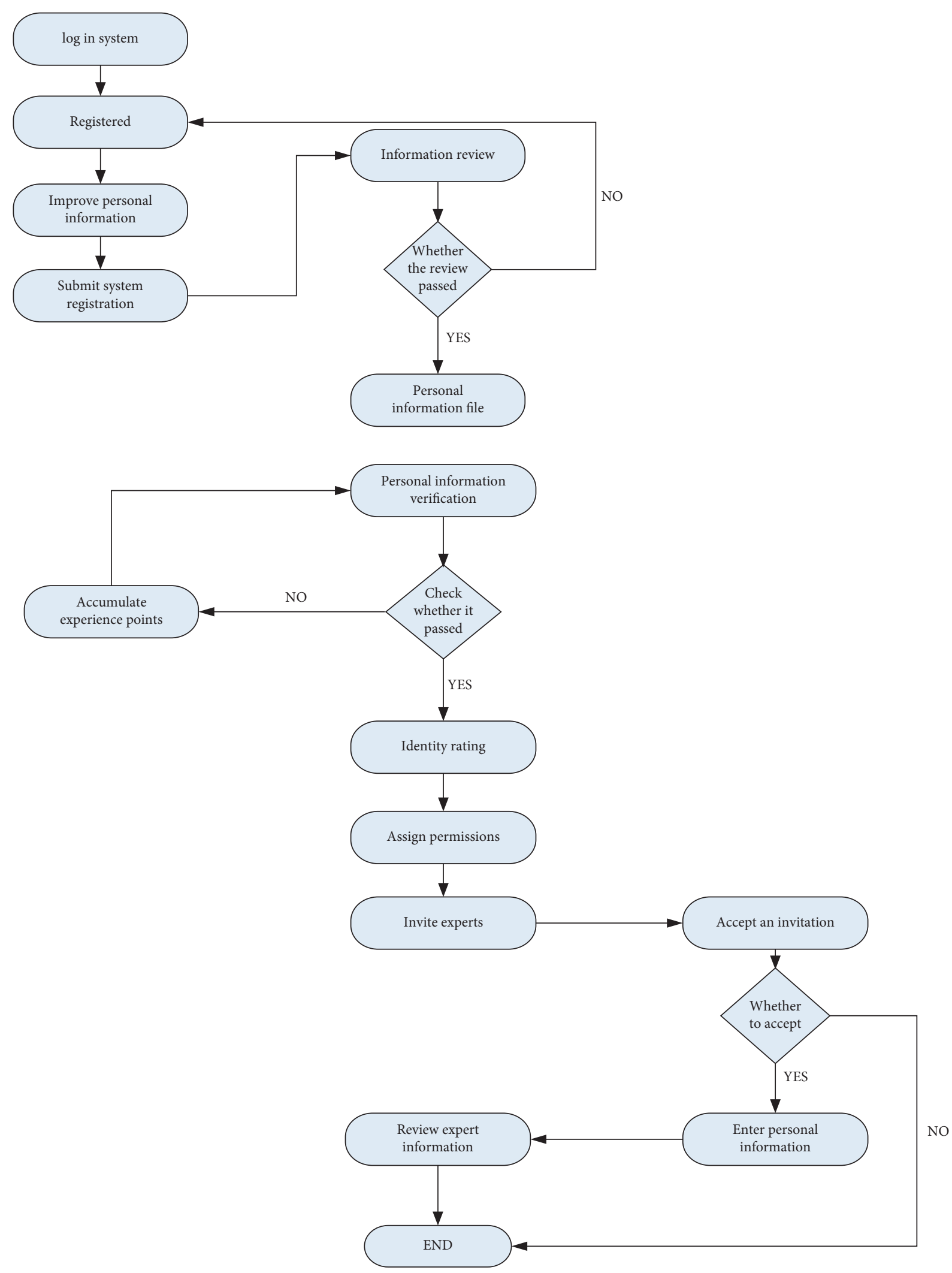

FIgURE 4: Flow chart of ceramic appreciation and exchange business management.

anticorrosive, and has a firm and strong tire quality, and the sound that it blows out is very fragile [25]. Ceramics are our country's exquisite handicrafts since ancient times and have been sold overseas and widely circulated. Our country's ceramics have always been loved by all parties at home and abroad, and it is a popular business card for our country in 
the world. Therefore, we designed and developed the system on this basis, using the new mobile edge computing technology to make a systematic summary of ceramic appreciation, communication, and management [26]. The mobile information platform is a platform designed and developed on the basis of modern mobile Internet technology, 5G, mobile communication technology, etc. It can communicate messages on multiple platforms such as mobile phones, tablets, and laptops to achieve management, work, and mobile information of service and communication which provides high-quality, fast, and convenient management services to all parties in the society. We use the latest technology to design and develop the system, which can make our traditional artworks well protected, and show their beauty to everyone in the world. This is the original intention of our system design and development [27].

\section{Data Analysis and Discussion}

4.1. Data Analysis Based on MEC. MEC is an emerging technology developed since the 2010s. To solve the above problems, large-scale mobile networks will have certain computing and storage capabilities. Capable small cells are placed at the edge of the mobile network, so how can these small cells scattered on the edge be used? For now, it is important to provide users with low latency and high bandwidth and reduce the workload on the backbone network. Therefore, the MEC provided by the ETSI is a promising computing model. This model is based on the evolutionary architecture of $5 \mathrm{G}$ networks and is proposed for the purpose of deep integration of Internet services and small cells. Based on a summary of the migration task initially uploaded by each user, including data size, load operation (that is, the number of CPU requirements each specific application requires for its work), local runtime, and expected output data size, when the mobile network receives this information, the global controller will prepare each migration job by assigning each migration job to a smaller base station, which can achieve the lowest delay when processing user migration tasks. Table 1 details the task information in mobile edge computing.

Figure 5 compares the number of data packets transmitted by the full-time channel trust protocol and CoCo. From Figure 5, we can see that the full-time channel trust protocol actually sends more data packets from CoCo, because both protocols will eventually send all data objects. The reason for the result is that the process is completed to make full use of the delivery time. The reliable host transfer protocol adds some XOR-encoded data packets to send more data packets, sending a fully active message. There is no need to increase energy consumption. This is because the power consumption of the device depends on the timing of the wireless signal. Therefore, the power consumption of transmission and data packet transmission is approximately the same during the transmission time.

The basic cloud data center and MEC system architecture is the core of the technology in this article. Phone users can access networks through a variety of wireless sources, such as mobile terminals and Wi-Fi access points. The MEC
Server data center is connected to the main cloud data center. When the heavy computing service from the mobile terminal application reaches the MEC server, the MEC server is divided into multiple tasks and runs in the core cloud data center to perform operations. This is because a data center in the cloud can be considered a "resource" that consists of many servers in the background. So it can run multiple tasks from the parallel MEC server. Therefore, there is no reason to consider queue delays when delegating secondary tasks to the primary server. This is because the current research on the MEC system architecture is considered theoretical, so we refer to the processing and caching capabilities of the micro cloud to realize the computing and caching capabilities of mobile terminals, MEC servers, and cloud data centers. Therefore, it is necessary to compare the delays of various factors in the appeal. See Table 2 for specific comparison details.

There are many differences between EMC and the current popular cloud computing. MEC add wireless processing and other functions to store and process to create an open platform for embedding applications. They enable wireless networks and services via wireless APIs, integrate wireless networks, and server services and upgrade traditional wireless base station to smart base station. In terms of handling business aspects such as the Internet of Things, short videos, medical rehabilitation, sales, etc., most modern laptops can provide customized and specialized services for the industry. This improves the efficiency of network use and adds value. At the same time, the development strategy of laptops (especially geographical location) can achieve the advantages of low latency and high bandwidth. The MEC can obtain more accurate real-time wireless network information and location information, thus providing a more accurate service. Cloud computing is based on current technologies such as computers, clouds, and 5G. Now, with the continuous development of science and technology, cloud computing is also a technology that is very much needed in all walks of life. Table 3 shows the difference data between MEC and cloud computing.

The current popular method is to run the actual application environment on the computer platform, find out and test the actual operating environment through the network platform, and connect to the base station through mobile devices to create and deploy the most advanced server services. When a mobile user uses a computer workflow, the workflow service can be downloaded to the top server through the mobile network. In the edge cloud, the edge server receives the mobile user's request and processes it when needed. Then, it returns to the mobile user through the mobile network. In this task, it is assumed that all services can be run locally or downloaded to the edge server. The research is to optimize the overall battery power consumption and the total time to complete the work. When mobile endpoint processes work in a mobile environment, we focus on smart algorithm design to improve efficiency for this problem. This is because many aspects need to be considered when optimizing these goals. In the optimization process, the calculation status of the limbs is first checked, and the final conclusion extends to the existence of many 
TABLE 1: Information about each task in mobile edge computing.

\begin{tabular}{cccccc}
\hline Task & $\begin{array}{c}\text { Data input size } \\
(\mathrm{MB})\end{array}$ & $\begin{array}{c}\text { Upload time/download } \\
\text { time }\end{array}$ & $\begin{array}{c}\text { Task and result delivery } \\
\text { time }\end{array}$ & $\begin{array}{c}\text { Small cell processing } \\
\text { time }(\mathrm{s})\end{array}$ & $\begin{array}{c}\text { Local processing time of user } \\
\text { equipment }(\mathrm{s})\end{array}$ \\
\hline$t 1$ & 35 & $3.5 \mathrm{~s}$ and $1.5 \mathrm{~s}$ & $0.35 \mathrm{~s}$ and $0.15 \mathrm{~s}$ & 20 & 110 \\
$t 2$ & 40 & $4.0 \mathrm{~s}$ and $1.6 \mathrm{~s}$ & $0.40 \mathrm{~s}$ and $0.16 \mathrm{~s}$ & 18 & 90 \\
$t 3$ & 40 & $4.0 \mathrm{~s}$ and $1.6 \mathrm{~s}$ & $0.40 \mathrm{~s}$ and $0.16 \mathrm{~s}$ & 15 & 60 \\
$t 4$ & 30 & $3.0 \mathrm{~s}$ and $1.4 \mathrm{~s}$ & $0.30 \mathrm{~s}$ and $0.14 \mathrm{~s}$ & 19 & 100 \\
$t 5$ & 25 & $2.5 \mathrm{~s}$ and $0.4 \mathrm{~s}$ & $0.25 \mathrm{~s}$ and $0.13 \mathrm{~s}$ & 14 & 50 \\
\hline
\end{tabular}

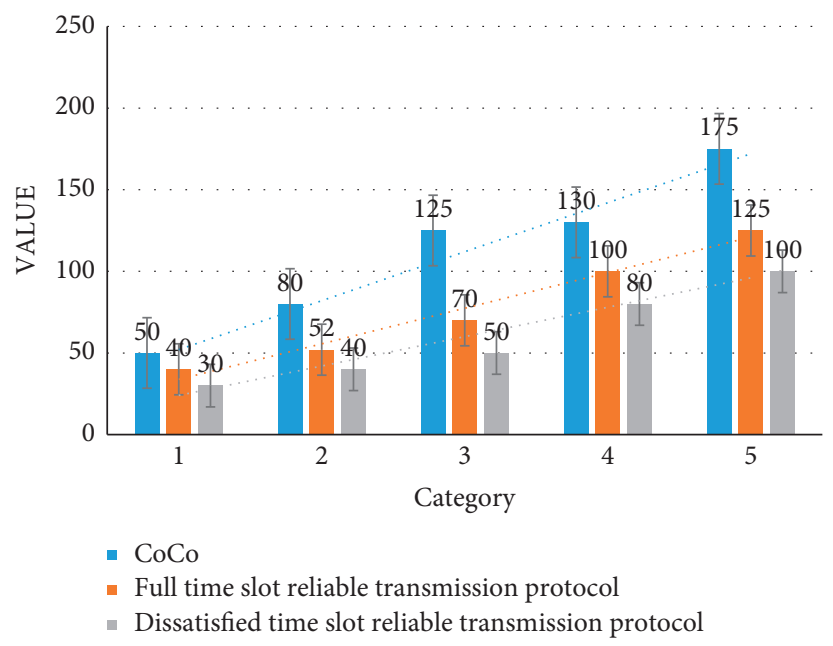

FIgURE 5: Experimental results of the test platform: transmission rounds.

TABLE 2: Delay comparison of various factors.

\begin{tabular}{lccc}
\hline & Mobile terminal & MEC server & Core cloud \\
\hline Calculate ability & 1 & $100-10 \mathrm{~K}$ & $10 \mathrm{~K}-100 \mathrm{M}$ \\
Storage capacity & 1 & 100 & $100 \mathrm{~K}-1 \mathrm{M}$ \\
Average delay & 1 & 10 & 1000 \\
\hline
\end{tabular}

TABle 3: The difference between EMC and cloud computing.

\begin{tabular}{lcc}
\hline Feature & Cloud computing & Mobile edge computing \\
\hline $\begin{array}{l}\text { Network } \\
\text { deployment }\end{array}$ & Centralized: the data processing center is located in & $\begin{array}{c}\text { Distributed: the server is located between the data center and } \\
\text { the terminal }\end{array}$ \\
User distance & the cloud & Close to the end user \\
Time delay & Har away from the end user & Low latency \\
Scalability & Higher latency & Center and network edge \\
Storage capacity & Data center only & Low \\
Power loss & High & Low \\
\hline
\end{tabular}

mobile devices and multiple mobile terminals, as shown in Figure 6.

The users of the ceramic appreciation and communication management system are mainly composed of four types of personnel: art lovers, art experts, and staff and backend managers. Art lovers: most art lovers are mainly concerned about art appreciation, but they also appreciate art consultation and education services, including sharing valuation and exchange activities. Art experts: in analyzing the initial business process, professionals are mainly responsible for signing and evaluating artworks. The art investment consultation includes lectures, public welfare training, and participation in art appreciation and exchange activities. Staff: for the analysis of traditional business processes, the organizer is mainly responsible for organizing and coordinating all activities such as art appreciation, communication, and training. Back-office management personnel: in the analysis of traditional business processes, 


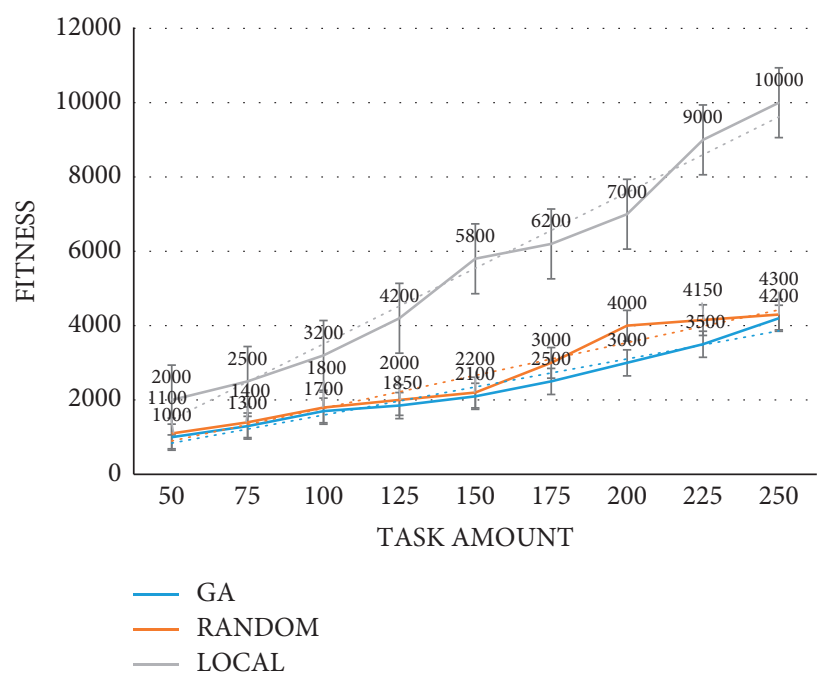

Figure 6: Comparison of several types of MEC.

TABLE 4: User role division table.

\begin{tabular}{lc}
\hline Character & Responsibilities or functions \\
\hline $\begin{array}{l}\text { Art lovers } \\
\text { Art expert }\end{array}$ & The general role of the system, and the main user of the system, performing various activities \\
Staff member & Ceramic art appreciation experts or related artists participate in appreciation, training, and exchanges \\
Backstage manager & Related staff guarantee the development and design of the system \\
The technicians ensure that the entire system is normal and some postwork
\end{tabular}

TABle 5: Specific details of art appreciation.

\begin{tabular}{|c|c|}
\hline Descriptive item & Description \\
\hline Use case name & Art appreciation \\
\hline The use case description & Describes the process of expert appraisal and appreciation of art \\
\hline Participants & Managers, experts \\
\hline Preconditions & Can read the art information and expert information \\
\hline Postconditions $(n)$ & Generate appreciation record information \\
\hline \multirow{5}{*}{ Basic operation flow } & Successfully log into the system platform \\
\hline & Set up the art crystal to be inspected \\
\hline & Designated appreciation experts \\
\hline & $\begin{array}{c}\text { Complete the authenticity, chronology, and artistic appreciation of artworks } \\
\text { Reinspection }\end{array}$ \\
\hline & Submit the assessment (appreciation) report \\
\hline
\end{tabular}

most of them are management art, collection of related materials, and post-system maintenance work. The specific roles involved in the ceramic appreciation and communication management system are shown in Table 4.

Porcelain is a priceless treasure in the Chinese tradition. It is usually priceless and exquisite. It combines ornamental value and practical value. It can be a container that people often use in daily life and can be used frequently in daily life. It can also be the artwork played by various merchant tycoons, which is invaluable and worth collecting. With people's understanding of art nowadays and investors' emphasis on porcelain, ceramic art has become a very familiar category in the art industry. The importance of ceramics is increasing day by day, and more and more attention is paid to the investment, appreciation, and protection of ceramics and other related works of art. The specific details of ceramic art appreciation are shown in Table 5.

As more and more domestic and foreign art lovers like our country's ceramic art, the porcelain we now fire in our daily life is also very popular abroad, such as sinks and bathtubs, which are also very common in foreign countries and well received. From 2016 to 2020, our country's export of ceramics has increased year by year, bringing high 


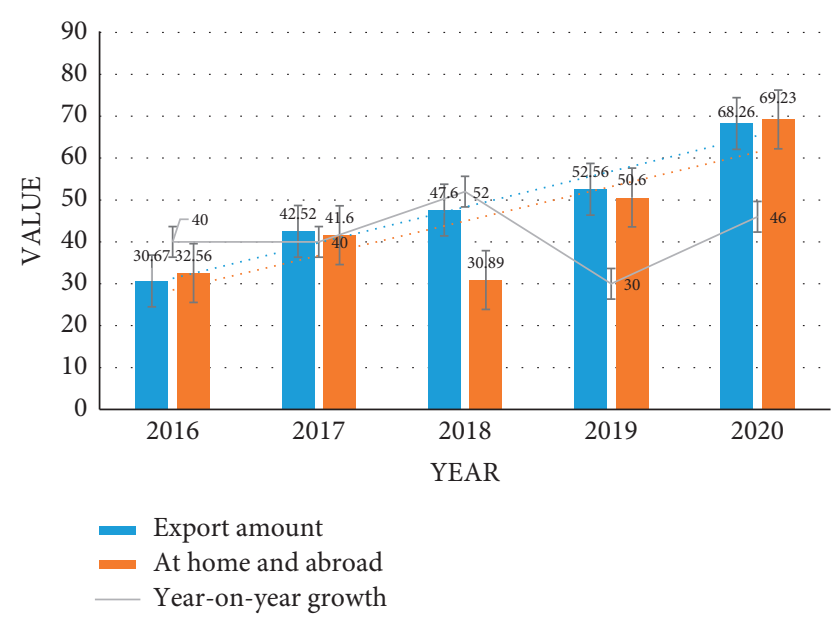

Figure 7: Export value and growth of our country's ceramic industry from 2016 to 2020 .

development to our country's ceramic industry, as shown in Figure 7.

4.2. Discussion. This section is based on the design and development with EMC and is used for the experimental part of the mobile information system for ceramic appreciation, communication, and management. The experiment part uses a variety of different technologies to explain the system in detail, and some of the specific experimental data are explained in detail through tables and diagrams. MEC is based on computer development of the $5 \mathrm{G}$ network architecture and is proposed for the purpose of deep integration of Internet services and small cells. Based on a summary of the migration task uploaded by each user at the beginning, including data size, load operation (that is, the number of CPU requirements each specific application requires for its work), local runtime, and expected output data size, using this technology in our traditional ceramic appreciation and communication management, combining the latest technology with our traditional culture, and using the system in our daily art exhibitions make the exhibition more systematic and organized.

\section{Conclusions}

The aim of this article is to design a mobile information system for ceramic appreciation, communication, and management, using the current popular mobile edge computing technology to design and develop the system. It allows users to experience a very high-quality network experience, such as speeding up network loading, service response, application downloading, and other functions. In general, mobile information is the realization of various information applications based on mobile terminals such as smart phones, tablets, smart watches, and technologies such as telecommunications and the Internet and finally allows you to use mobile devices around you at any time. Combining these with traditional ceramics in our country, the mobile information system for ceramic appreciation, communication, and management designed and developed is now in line with the current development. Although our system is still at the theoretical level, we must develop it. It will be widely used in the ceramic industry for a long time in the future.

\section{Data Availability}

No data were used to support this study.

\section{Conflicts of Interest}

The author declares no conflicts of interest.

\section{Authors' Contributions}

The author has read the manuscript and approved it for submission.

\section{References}

[1] T. X. Tran, A. Hajisami, P. Pandey, and D. Pompili, "Collaborative mobile edge computing in 5G networks: new paradigms, scenarios, and challenges," IEEE Communications Magazine, vol. 55, no. 4, pp. 54-61, 2017.

[2] L. Liu, C. Xin, Z. Lu, L. Wang, and X. Wen, "Mobile-edge computing framework with data compression for wireless network in energy internet," Tsinghua Science \& Technology, vol. 24, no. 3, pp. 271-280, 2019.

[3] Y. Jararweh, M. Al-Ayyoub, M. Al-Quraan, L. Tawalbeh, and E. Benkhelifa, "Delay-aware power optimization model for mobile edge computing systems," Personal \& Ubiquitous Computing, vol. 21, no. 6, pp. 1-11, 2017.

[4] P. Wumin and M. Rui, "Research on improvement of China mobile customer relationship management system based on apriori algorithm," Boletin Tecnico/Technical Bulletin, vol. 55, no. 19, pp. 520-526, 2017.

[5] S. Henriques and M. J. Damasio, "The value of mobile communication for social belonging: mobile apps and the impact on social interaction," International Journal of Handheld Computing Research, vol. 7, no. 2, pp. 44-58, 2016.

[6] S. Wan, L. Qi, X. Xu, C. Tong, and Z. Gu, "Deep learning models for real-time human activity recognition with smartphones," Mobile Networks and Applications, vol. 25, no. 1, pp. 743-755, 2019.

[7] Z. Wen and Lu, "Research and implementation of computer network user behavior forensics system based on system a log," Journal of Information Security Research, vol. 9, no. 1, pp. 23-40, 2018.

[8] H. Du and Y. Guang, "Analysis and research on SPSS clustering of mobile communication data under mobile signal interference," Boletin Tecnico/Technical Bulletin, vol. 55, no. 8, pp. 210-217, 2017.

[9] C. Vallati, A. Virdis, E. Mingozzi, and G. Stea, "Mobile-edge computing come home connecting things in future smart homes using LTE device-to-device communications," IEEE Consumer Electronics Magazine, vol. 5, no. 4, pp. 77-83, 2016.

[10] Y. Wang, S. Min, X. Wang, L. Wang, and J. Li, "Mobile-edge computing: partial computation offloading using dynamic voltage scaling," IEEE Transactions on Communications, vol. 64, no. 10, pp. 4268-4282, 2016.

[11] T. Taleb, S. Dutta, A. Ksentini, M. Iqbal, and H. Flinck, "Mobile edge computing potential in making cities smarter," IEEE Communications Magazine, vol. 55, no. 3, pp. 38-43, 2017.

[12] T. Q. Dinh, J. Tang, Q. D. La, and T. Q. S. Quek, "Offloading in mobile edge computing: task allocation and computational 
frequency scaling," IEEE Transactions on Communications, vol. 65, no. 8, pp. 3571-3584, 2017.

[13] C. Wang, C. Liang, F. R. Yu, Q. Chen, and L. Tang, "Computation offloading and resource allocation in wireless cellular networks with mobile edge computing," IEEE Transactions on Wireless Communications, vol. 16, no. 8, pp. 4924-4938, 2017.

[14] Z. Ke, Y. Mao, S. Leng, Y. He, and Y. Zhang, "Mobile-edge computing for vehicular networks: a promising network paradigm with predictive off-loading," IEEE Vehicular Technology Magazine, vol. 12, no. 2, pp. 36-44, 2017.

[15] D. Sabella, A. Vaillant, P. Kuure, U. Rauschenbach, and F. Glust, "Mobile-edge computing architecture: the role of MEC in the internet of things," IEEE Consumer Electronics Magazine, vol. 5, no. 4, pp. 84-91, 2016.

[16] S. Ivanaj, G.-B. Nganmini, and A. Antoine, "Measuring E-learners' perceptions of service quality," Journal of Organizational and End User Computing, vol. 31, no. 2, pp. 83-104, 2019.

[17] B. P. Rimal, D. P. Van, and M. Maier, "Mobile-edge computing empowered fiber-wireless access networks in the $5 \mathrm{G}$ era," IEEE Communications Magazine, vol. 11, no. 2, pp. 192-200, 2016.

[18] X. Lyu, W. Ni, H. Tian et al., "Optimal schedule of mobile edge computing for internet of things using partial information," IEEE Journal on Selected Areas in Communications, vol. 35, no. 11, pp. 2606-2615, 2017.

[19] F. Zhou, Y. Wu, R. Q. Hu, and Y. Qian, "Computation rate maximization in UAV-enabled wireless-powered mobileedge computing systems," IEEE Journal on Selected Areas in Communications, vol. 36, no. 9, pp. 1927-1941, 2018.

[20] X. Huang, R. Yu, J. Kang, Y. He, and Y. Zhang, "Exploring mobile edge computing for 5G-enabled software defined vehicular networks," IEEE Wireless Communications, vol. 24, no. 6, pp. 55-63, 2018.

[21] X. Lyu, H. Tian, L. Jiang et al., "Selective offloading in mobile edge computing for the green internet of things," IEEE Network, vol. 32, no. 1, pp. 54-60, 2018.

[22] K. Zhang, S. Leng, Y. He, S. Maharjan, and Y. Zhang, "Mobile edge computing and networking for green and low-latency internet of things," IEEE Communications Magazine, vol. 56, no. 5, pp. 39-45, 2018.

[23] I. W. Mudra, I. G. M. Raharja, and I. W. Sukarya, "Estetika visual kriya keramik berornamen wayang khas bali," Gondang: Jurnal Seni dan Budaya, vol. 5, no. 1, pp. 53-63, 2021.

[24] N. Inácio, F. Nocete, J. M. Nieto, and M. R. Bayona, "Pottery and metallurgical activity in southwest of the iberian peninsula during III milennium B.C.E.: technological choices in the manufacture of ceramic instruments associated to the metallurgical process," Complutum, vol. 28, no. 1, pp. 51-69, 2017.

[25] Z. Lv, Z. Tan, W. Qian, and Y. Yang, "Cloud computing management platform of human resource based on mobile communication technology," Wireless Personal Communications: International Journal, vol. 102, no. 2, pp. 1-14, 2018.

[26] G. Abdelkader, H. Said Naima, and A. P. Adda, "Secure authentication approach based new mobility management schemes for mobile communication," Journal of Information Processing Systems, vol. 13, no. 1, pp. 152-173, 2017.

[27] A. Aldini, J.-M. Seigneur, C. Ballester Lafuente, X. Titi, and J. Guislain, "Design and validation of a trust-based opportunity-enabled risk management system," Information \& Computer Security, vol. 25, no. 1, pp. 2-25, 2017. 